\title{
Uncomplicated COVID-19 disease of an adolescent with pre-existing pulmonary condition
}

\author{
Franziska Fischer ${ }^{1}$, Anne Daun ${ }^{1}$, and Sebastian Becker ${ }^{1}$ \\ ${ }^{1}$ Darmstadt Hospital
}

July 28, 2020

To the editor,

we describe a 12-year-old boy with uncontrolled bronchial asthma, bronchiolitis obliterans and restrictive and obstructive pulmonary disorders in spirometry who became infected with COVID-19. Contrary to the assumptions of other clinical findings, the boy was complaining only one day about body aches while showing no other symptoms. Neither cough, dyspnoea nor fever occurred. This case demonstrates that in particular paediatric patients with pre-existing pulmonary condition such as uncontrolled bronchial asthma and bronchiolitis obliterans can show an uncomplicated course of the COVID-19 disease.

As often-described in literature, severe clinical courses of the COVID-19 disease happen to occur in patients with pre-existing conditions such as: heart or lung diseases, cancer, diabetes, high blood pressure or among the elderly (1). Some young patients even had been previously healthy but then showed a severe progress of a COVID-19 infection (2).

In spring 2015, the - at the time - 7-year-old boy was admitted to the hospital for the first time because of respiratory insufficiency. He was receiving oxygen as primary treatment. Even then after his first admission to the hospital the spirometry showed an obstructive and a restrictive pattern (vital capacity (VC): $61.3 \%$, forced expiratory volume in one second (FEV1): 75.4\%, FEV1/VC ratio: 100\%). Consequently, based on the results, an inhalation with corticosteroid (ICS)/long-acting $\beta_{2}$-agonist (LABA) combination was chosen to start the therapy. An early bloomer allergy also was found, which caused a rhino conjunctivitis during pollen season. A bronchiolitis obliterans, which was diagnosed by a CT scan (see Fig.1) is mostly caused by a mycoplasma infection. Unfortunately, there was no improvement shown after the treatment with azithromycin and methylprednisolone. Since there was no positive effect on the pulmonary situation after starting a treatment with Leukotriene Receptor Antagonists (LTRAs), a treatment with formoterol was initiated. Bronchoscopy in March 2019 showed a chronic purulent bronchitis, but no pathogen was detected.

Last January, the boy was admitted to the hospital to control the success of the therapy because inpatient treatment possibilities were exhausted. Formoterol was ended and an inhalative therapy with tiotropium bromide was started.

During the COVID-19 pandemic on April 15th the 12-year-old boy presented himself in our paediatric pneumological consultation with still uncontrolled asthma bronchial (GINA severity IV), bronchiolitis obliterans and currently mild allergic rhino conjunctivitis. The obese boy (weight $69.3 \mathrm{~kg}$, height $161.1 \mathrm{~cm}$ ) reported that he coughed dry daily both during the day and at night. During physical exertion, the cough increased. The current medication Salmeterol/Fluticason 25 $\mathrm{g} / 250 \mu \mathrm{g}$ 1-0-1 stroke, LTRA 5mg, tiotropium bromide $5 \mu \mathrm{g}$ was taken daily. He had not had to inhale salbutamol since the last performance in our consultation in February. A slight wheezing was found in the upper lobes of the lungs. Blood results showed a lymphocytosis $(65 \%)$ and neutropenia (23\%) and a normal blood count of leukocytes $(4.3 / \mathrm{nl})$, there was also found a microcytic hypochromic anaemia (Hb 11.3g/dl, Hct 0.35l/l, MCV 78f, MCH, 25pg, MCHC 32g/dl). The spirometry 
showed an obstructive and restrictive ventilation disorder (see Fig. 2, (VC): 48\%, (FVC): 58\%, (FEV1): 54\%, FEV1/VC: 77.85\%, (PEF):66\%, FEF 25: 69\%, FEF 50 43\%, FEF 75 27\%). The bronchodilation test was negative (see Fig. 3).

The following day, the mother of our patient developed fever as well as head and limb pain. Since the mother had been in contact with a COVID-19 patient as a nurse in a nursing home, she was also tested positive for coronavirus SARS-CoV-2 on April 17th 2020. The same day, the 12-year-old boy complained of limb pain. The COVID-19 PCR swab test was also found positive in him, his father and his sister who all live in the same household. During the quarantine period (14 days) and beyond, there were no further symptoms in the boy, he did not cough more than before, had no dyspnoea or fever and no need to use his asthma spray salbutamol.

After 24 days, the COVID-19 smear of our patient finally was negative. Spirometry still showed an obstructive and restrictive pattern, but some better values: (VC): 63\%, (FEV): 64\%, (FEV1): 60\%, (FEV1/VC): 77.66\%, Peak-Flow (PEF): 57\%, FEF 25: 64\%, FEF 50 48\%, FEF 75 32\%).

The WHO pronounces "older people and people of all ages with pre-existing medical conditions (such as diabetes, high blood pressure, heart disease, lung disease, or cancer) appear to develop serious illness more often than others" (1). Our 12-year-old patient who has two different lung diseases, bronchiolitis obliterans and a bronchial asthma, which was uncontrolled at the time, contracted the coronavirus. The boy showed no typical symptoms of a COVID-19 infection such as fever, dry cough or tiredness. He did not have to use his asthma spray salbutamol because of dysponea. He only complained about body aches for one day. Interestingly, the COVID-19 smear was positive for SARS-CoV-2 for more than 3 weeks. In some cases, viral genetic material could be detected up to 42 days after the onset of symptoms (4). Inhalation steroids may be the cause of prolonged virus detection, but there is currently no data on this in literature. Last but not least, it is interesting that the boy did not infect any other people with the coronavirus even before being diagnosed, for example in our consultation.

This case demonstrated that even a patient with uncontrolled bronchial asthma and furthermore with bronchiolitis obliterans is able to have an asymptomatic COVID-19 infection.

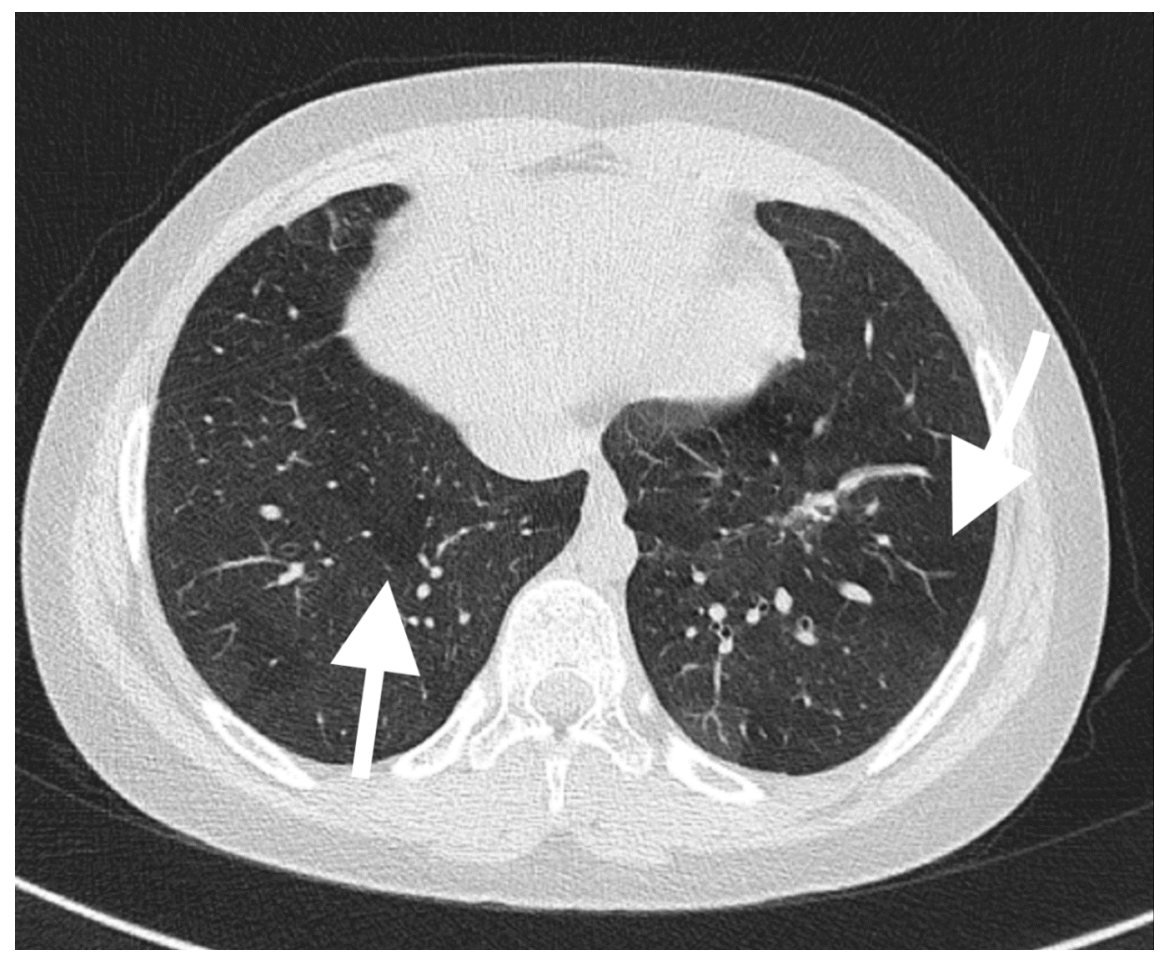


Fig. 1 HRCT of the 12-year- old boy: focal airtrapping (arrows).
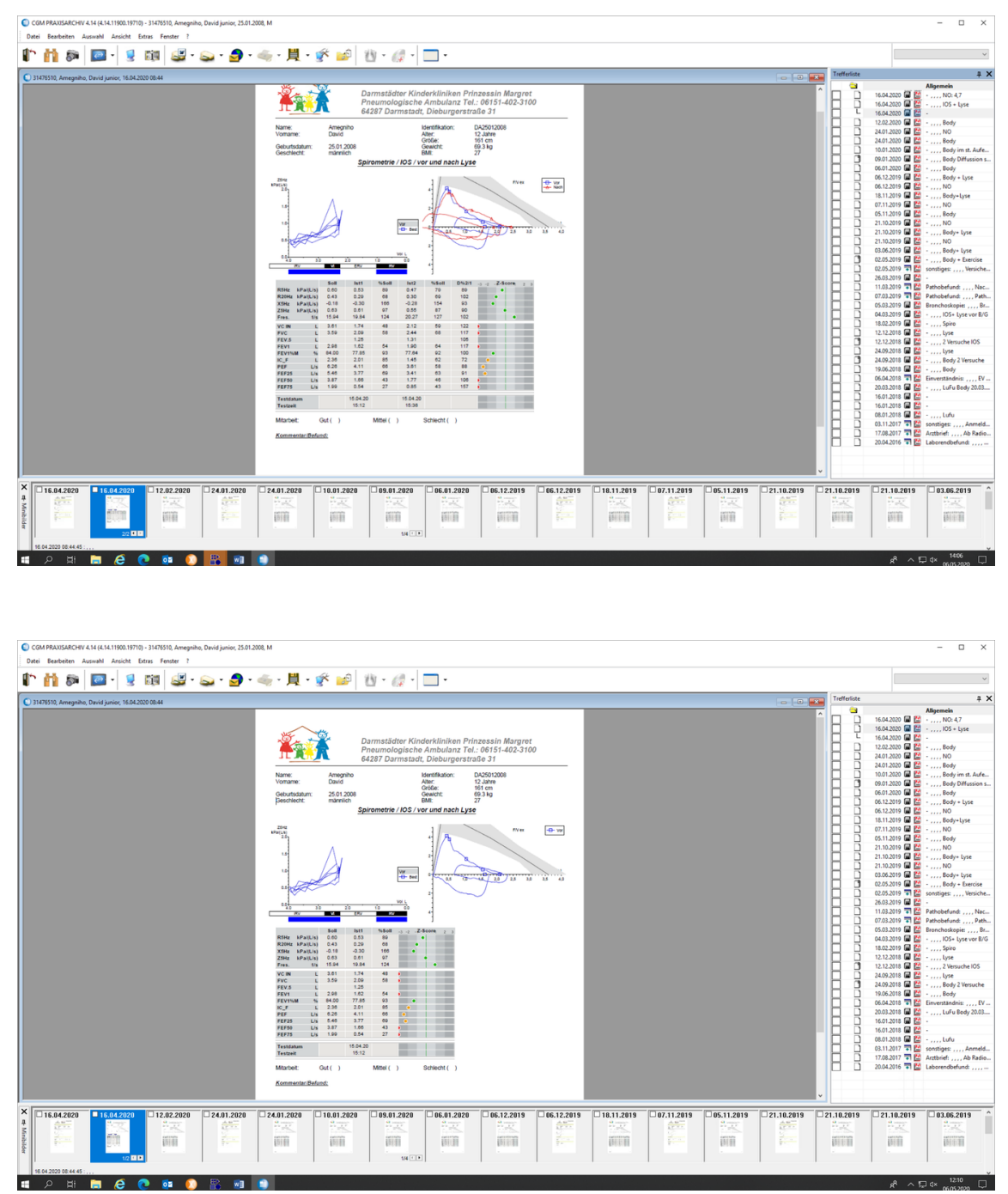

Fig. 2 Spirometry Fig. 3 Spirometry and bronchodilation test

\section{References}

1. https://www.who.int/emergencies/diseases/novel-coronavirus-2019/question-and-answers-hub/q-adetail/q-a-on-on-covid-19-for-older-people

2. Wee S.-L, Wang V 2020 https://www.nytimes.com/interactive/2020/03/13/world/asia/coronavirusdeath-life.html

3. https://www.abc.net.au/news/2020-04-23/coronavirus-test-positive-second-time-how-long-viruslasts/12175972

4. https://www.rki.de/SharedDocs/FAQ/NCOV2019/gesamt.html 